София, 2020. Sofia

\title{
Heat Flow and Seismicity in Bulgaria
}

Mikhail Khutorskoy', Emil Botev ${ }^{2}$, Valentina Protopopova ${ }^{2}$, Aleksey Benderev $^{3}$, Elena Tevelova ${ }^{1}$

${ }^{1}$ Geological Institute, Geological Institute of the Russian Academy of Sciences, Moskva, Russia; e-mail: mkhutorskoy@ginras.ru

${ }^{2}$ National Institute of Geophysics, Geodesy and Geography, Bulgarian Academy of Sciences, 1113 Sofia, Bulgaria, e-mail: ebotev@geophys.bas.bg;

${ }^{3}$ Geological Institute, Bulgarian Academy of Sciences, 1113 Sofia, Bulgaria; e-mail: alekseybenderev@yahoo.com

\section{Топлинен поток и сеизмичност в България}

\author{
Михаил Хуторской, Емил Ботев, Валентина Протопопова, Алексей \\ Бендерев, Елена Тевелева
}

Khutorskoy, M., E. Botev, V. Protopopova, A. Benderev, E. Tevelova. 2020. Heat Flow and Seismicity in Bulgaria. Engineering Geology and Hydrogeology, 34, 53-65.

\begin{abstract}
The study of intra-earth processes requires a comprehensive approach, which should be based on the understanding different nature and origin depth of terrestrial processes. In this regard, the problem of the relationship between seismicity and heat flow in the lithosphere is of interest. We analyzed seismic observation data in Bulgaria from 1981 to 2018 using statistical methods. A significant correlation was found between the heat flow and the earthquakes magnitude, as well as between the thickness of thermal lithosphere and the magnitude. To assess the released friction heat and hydrodynamic processes during the earth's crust movements, it is necessary to develop theoretical models based on a comprehensive analysis of the geological structure and geophysical fields in seismically active regions.
\end{abstract}

Keywords: seismicity, magnitude, heat flow, lithosphere, correlation, Bulgaria.

Резюме. Изследването на процесите, протичащи в Земята, изисква прилагането на комплексни подходи, които да се основават на познаването и установяването на връзка между различни по генезис и характер процеси. В това отношение интересен проблем е изясняването на връзката между сеизмичността и топлинното поле в земната кора. За целта са анализирани данни от мониторинга на сеизмичните явления в България за периода от 1981 до 2018 година, като са използвани статистически подходи. Установена е значима корелация между топлинния поток и магнитудите на земетресенията, а така сьщо и между дебелината на термичната литосфера и магнитудите. Подчертана е необходимостта от разработването на теоретични модели за изчисляване на стойността на отделената фрикционна топлинна енергия при придвижване на блоковете на земната кора, а също така и за хидродинамичните явления, като предвестник на земетресенията.

Ключови думи: сеизмичност, магнитуд, топлинен поток, литосфера, корелация, България. 


\section{Въведение}

Изследването на процесите, протичащи в Земята, изисква прилагането на комплексни подходи, които да се основават на познаването и установяването на връзка между различни по генезис и характер процеси. В това отношение интересен проблем е изясняването на връзката между сеизмичността и топлинното поле в земната кора. Този въпрос за България е разгледан и публикуван в Русия от Хуторской и др. (Khutorskoy et al., 2019a,b), но този материал е труднодостъпен за заинтересуваните специалисти в България. Затова, цел на настоящата работа е да се представят получените основни резултати от това изследване пред българската научна общност. България попада в обсега на един от най-мощните геодинамични пояси на планетата Алпо-Хималайския. В него, заедно с Тихоокеанския пояс (Circum-Pacific Belt), се случват 1 милион земетресения, което е средно 120 труса в час или два за минута. Често по-силните земетресения обикновено се съпровождат от други катастрофални процеси: цунами, срутища, свлачища, кални потоци и т.н., което налага държавите, разположени в пределите на сеизмично опасни региони, да обръщат сериозно внимание на сеизмичния мониторинг. Усъвършенстването на методите на мониторинга на природните катастрофи се провежда постоянно. Ефективността на прогнозата в немалка степен зависи от наличната апаратурна база, т.е. от точността на датчиците, методиката на разположението им, честотата на измерванията, начина на предаване на информацията и скоростта на обработка на получените данни. Освен чисто апаратурно-методологичните изисквания, развитите държави оборудват активни геодинамични полигони, и разбира се повишават вероятността на прогноза за сеизмичните събития.

За повишаване на достоверността на прогнозирането, освен усъвършенстване на апаратурната база, важен раздел от работата се явяват новите методични разработки, изчисляването на модели на дълбочинните геодинамични процеси, създаването на програмни средства за обработка на информацията, търсенето на корелационни връзки между различните геоложки, геохимични и геофизични проявления. За формирането на система от сеизмопрогнозни наблюдения е необходимо наличието на регионална характеристика на разпространението на огнищата на земетресения и други ендогенни прояви във вид на карти, каталози, монографии, исторически хроники, описание на хода на една или друга природна катастрофа, а така също и данни за човешки и материални загуби. Цялата тази информация е необходима за оценяване на степента на природния и социалния сеизмичен риск, за създаване на нормативи за сеизмоустойчиво строителство на дадена територия, за подготовка на евакуационно оборудване и на специализирани спасителни отряди.

\section{Изследване на статистическата връзка между сеизмичните и геотермичните параметри}

В настоящото проучване е изследвана връзката между магнитуда на сеизмичните събития на територията на България и плътността на топлинния поток. Априорно може да се твърди, че тези два геофизични параметьра са свързани генетично. Земетресенията възникват при разкъсване на целостта на средата, т.е. в крехък относително студен блок, в който трябва да се наблюдава относително намаляване на температурата, съпровождано от нисък топлинен поток. Ако температурата и топлинния поток в литосферата са високи, то скалите стават по-пластични и при увеличаване на напрежението постепенно се деформират без разкъсване на скалите. В най-негативния случай това може да доведе до незначителни по магнитуд сеизмични прояви.

Във връзка с гореказаното, анализът на регионалното разпределение 
на топлинния поток, а особено на зоните с резки хоризонтални промени на термичните характеристики, може да бъде прогнозен признак за оценка на сеизмичния риск за един или друг блок на литосферата (Khutorskoy et al., 1999).

Местоположението на България в северния фланг на Алпо-Хималайския пояс, предопределя присъствието на сеизмичен риск, и затова е оправдано провеждането на изследвания, насочени към дългосрочно и средносрочно прогнозиране на земетресения в режим на мониторинга на геофизичните полета (Botev et al, 2013). Съгласно данните за земетресения за 30 годишен период (фиг.1) (Aleksandrova et al., 1992, 1993; Solakov, Simeonova, 1993; Botev et al., 1993 - 2000, 2002, 2003, 2005, 2007, 2009 - 2013) се установява, че за този период не са се случвали големи разрушителни земетресения с $\mathrm{M}>6$, въпреки, че в историчното минало са известни катастрофални сеизмични събития, довели до промяна в релефа и речните русла. Най-сеизмоактивните на територията на страната са Старопланинският нагънат пояс, особено неговата източна, Причерноморска част, а така също и Родопският срединен масив.

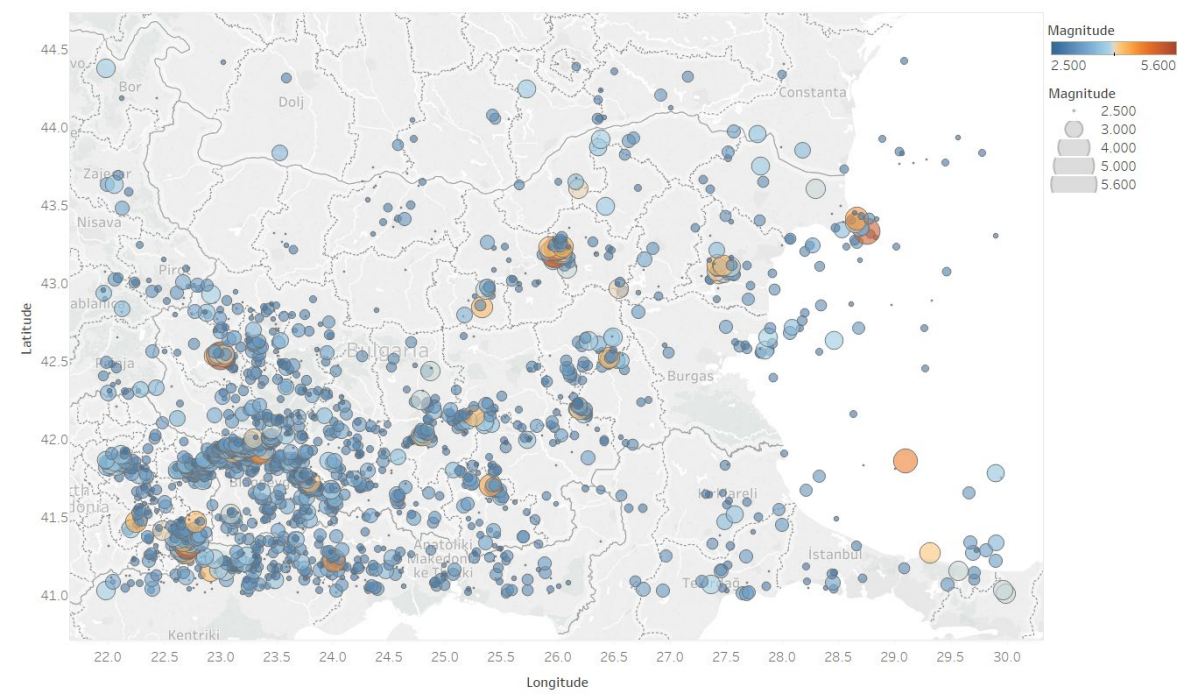

Фиг.1. Карта на разпределение на епицентрите на земетресенията според магнитудите им на територията на България и съседни територии за периода 1981-2012 г.

Fig. 1. Map of distribution of the epicenters of the earthquakes according to their magnitudes on the territory of Bulgaria and neighboring territories for the period 1981-2012.

Извършеният анализ на дълбочините на земетресенията за същия период на наблюдения показва, че най-дълбокофокусните събития са разположени в районите на Мизийската плоча, в пределите на България и Румъния, и в прилягащата им акватория на Черно море. Тази закономерност се обяснява от позицията на разпределението на топлинния поток, който в страната намалява от юг на север (Khutorskoy et al., 2019a,b). Ако се пресметнат дълбочинните температури в литосферата на България, изхождайки от топлофизичните модели стойностите на топлинния поток, излиза, че литосферата е повече нагрята в района на Родопския масив, и там преходът от крехко към пластично състояние на веществото се извършва на по-малка дълбочина, отколкото в районите на Мизийската плоча и Черноморската падина.

Проверката на статистическата значимост на корелацията на топлинния поток и магнитуда на земетресенията се осъществява въз основа на критерия на 
Пирс. Направено е сравнение между средните стойности на топлинния поток и магнитудите по квадрати $1^{\circ} \times 1^{\circ}$ (Табл.1). Емпиричната стойност на критерия е $-0,48$.

Таблица 1. Данни за изчисляване на корелацията между топлинния поток и магнитудите на земетресенията в България.

Table 1. Data for calculating the correlation between heat flux and earthquake magnitudes in Bulgaria.

\begin{tabular}{|c|c|c|c|}
\hline $\begin{array}{l}\text { геогр. ширина }\left(^{\circ}\right) \text { на } \\
\text { центъра на квадрата }\end{array}$ & $\begin{array}{l}\text { геогр. дължина }\left(^{\circ}\right) \text { на } \\
\text { центъра на квадрата }\end{array}$ & $\begin{array}{l}\text { средна стойност на } \\
\text { топлинния поток }(q) \\
\text { в квадрата, mW/m }{ }^{2}\end{array}$ & $\begin{array}{l}\text { средна стойност } \\
\text { на магнитуда }(M) \text { в } \\
\text { квадрата }\end{array}$ \\
\hline 43,5 & 24,5 & 92 & 2,50 \\
\hline 43,5 & 26,5 & 72 & 2,94 \\
\hline 41,5 & 24,5 & 75 & 3,04 \\
\hline 41,5 & 23,5 & 77 & 3,05 \\
\hline 41,5 & 25,5 & 74 & 3,17 \\
\hline 42,5 & 25,5 & 66 & 3,20 \\
\hline 42,5 & 24,5 & 62 & 3,28 \\
\hline 43,5 & 22,5 & 65 & 3,40 \\
\hline 42,5 & 23,5 & 65 & 3,49 \\
\hline 43,5 & 25,5 & 76 & 3,53 \\
\hline 42,5 & 26,5 & 59 & 3,68 \\
\hline 42,5 & 27,5 & 54 & 3,95 \\
\hline 42,5 & 22,5 & 63 & 4,12 \\
\hline 43,5 & 27,5 & 83 & 4,20 \\
\hline
\end{tabular}

Статистическата значимост на емпиричния коефициент на корелацията на Пирс $\left(t_{\text {емп }}\right)$ се определя по формулата:

$$
t_{\text {емп }}=\frac{|r| \sqrt{n-1}}{1-r^{2}}
$$

Ако тази стойност е по-висока от критичната стойност на $t$-критерия на Стюдънт, при степен на свобода $k=n-2$ и с ниво на значимост (1- $\alpha)$, то тогава коефициентът на корелация $(r)$ е значим. В случая $\mathrm{r}=-0,48, n=14, k=12$, а $t_{\text {емп }}=2,248$. Критичната стойност на $t$-критерия на Стюдънт при брой на степените свобода и доверителна вероятност $P=0,95$ е равна на 2,179. Следователно, коефициентът на корелация се приема за значим.

Регресионната зависимост между магнитуда на земетресенията $(M)$ и топлинния поток $\left(q, \mathrm{~mW} / \mathrm{m}^{2}\right)$ е дадена на фиг. 2. Апроксимацията на линейната зависимост на тези параметри е представена с пунктирна линия. Тя се описва с формулата: $M=5,85-0,035 \cdot q$.

Стойностите на топлинния поток, строежът и дебелината на кората, а така също от топлопроводимостта на отделните слоеве на кората, определена въ3 основа на корелацията ѝ с граничните скорости на сеизмичните вълни (Hosney, 2000; Rybach, Buntebarth, 1984), са основа за оценката на дебелината на „термическата“ литосфера. Тя се асоциира с дълбочинното разположение на изотермата на солидуса на мантийното вещество. За континенталната литосфера, отчитайки РТ-условията на фазовия преход от твърдо към фракционно разтопено сьстояние, тази температура може да се приеме за $1200^{\circ} \mathrm{C}$. 


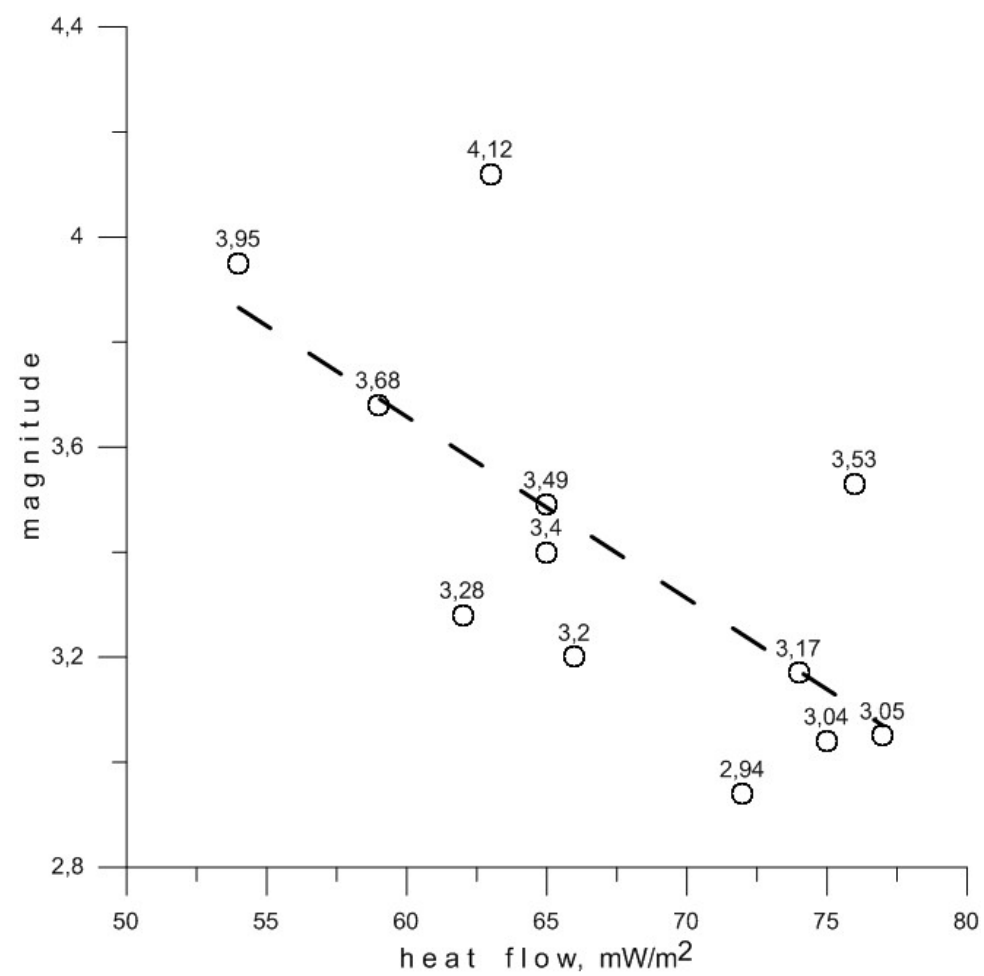

Фиг. 2. Корелационна връзка между топлинния поток и магнитуда на земетресенията (средни стойности по квадрати $1^{\circ} \times 1^{\circ}$ )

Fig. 2. Correlation between heat flow and magnitude of earthquakes (mean values per square $1^{\circ} \times 1^{\circ}$ )

На фиг. 3 е показана карта на пресметнатата дебелина на литосферата, т.е. разположението на дълбочината на тази изотерма. С най-малка дебелина на литосферата се характеризира Балканидната нагъната система и Мизийската плоча (по-малка от $150 \mathrm{~km}$ ), а с най-голяма - Родопският масив и система от грабени (Загорски, Тракийски и др.) (170-220 km), т.е. региони на България, които се характеризират с най-голяма степен на сеизмична активност (фиг. 3). За проверка на достоверността на твърдението за наличие на връзката между дебелината на литосферата $(L)$ и сеизмичната активност $(M)$ е извършен статистически анализ, след сравнение на съпоставимите бази от данни по квадрати $1^{\circ} \times 1^{\circ}$ (табл. 2).

За малки извадки $(n<20)$ за пресмятане на корелацията се използва коефициентът на рангова корелация на Спирмън.

За определяне на коефициента на рангова корелация на Спирмън предварително се провежда ранжиране по всеки от признаците (Табл. 3).

Коефициентът на рангова корелация на Спирмън ( $\rho)$ се определя по формулата:

$$
\rho=1-\frac{6 \sum d_{i}^{2}}{n\left(n^{2}-1\right)}
$$

където $d_{i}$ - разлика на ранговете на признаците (табл. 4). 


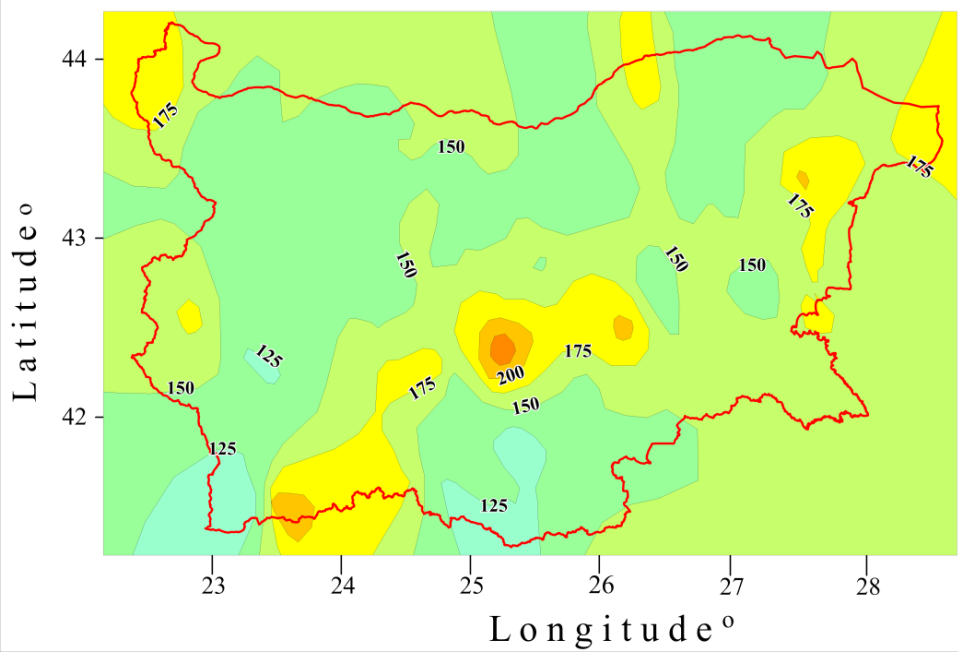

Фиг. 3. Карта на дебелините на термичната литосфера (положение на изотерма $1200^{\circ} \mathrm{C}$ ) на територията на България.

Fig. 3. Map of the thicknesses of the thermal lithosphere (position of the isotherm $1200^{\circ} \mathrm{C}$ ) on the territory of Bulgaria.

Таблица 2. Данни за изчисляване на корелацията между дебелината на литосферата $(L)$ и сеизмичната активност $(M)$

Table 2. Data for calculating the correlation between lithosphere thickness $(L)$ and seismic activity $(M)$

\begin{tabular}{|c|c|c|c|c|}
\hline $\begin{array}{l}\text { пореден номер } \\
\text { на квадрата }\end{array}$ & $\begin{array}{l}\text { географ. } \\
\text { ширина }\left(^{\circ}\right) \text { на } \\
\text { центъра на } \\
\text { квадрата }\end{array}$ & $\begin{array}{l}\text { географ. } \\
\text { дължина () } \\
\text { на центъра на } \\
\text { квадрата }\end{array}$ & $\begin{array}{l}\text { средна стойност } \\
\text { на дебелината на } \\
\text { литосферата }(L) \text { в } \\
\text { квадрата, } \mathrm{km}\end{array}$ & $\begin{array}{l}\text { средна стойност } \\
\text { на магнитуда } \\
(M) \text { в квадрата }\end{array}$ \\
\hline 1 & 43,5 & 22,5 & 161 & 2,84 \\
\hline 2 & 43,5 & 23,5 & 141 & 2,88 \\
\hline 3 & 43,5 & 24,5 & 144 & 2,92 \\
\hline 4 & 43,5 & 25,5 & 139 & 2,91 \\
\hline 5 & 43,5 & 26,5 & 149 & 2,73 \\
\hline 6 & 43,5 & 27,5 & 160 & 2,75 \\
\hline 7 & 43,5 & 28,5 & 180 & 2,82 \\
\hline 8 & 42,5 & 22,5 & 168 & 2,87 \\
\hline 9 & 42,5 & 23,5 & 134 & 2,60 \\
\hline 10 & 42,5 & 24,5 & 159 & 2,90 \\
\hline 11 & 42,5 & 25,5 & 176 & 2,81 \\
\hline 12 & 42,5 & 26,5 & 162 & 2,85 \\
\hline 13 & 42,5 & 27,5 & 165 & 2,70 \\
\hline 14 & 41,5 & 23,5 & 139 & 2,84 \\
\hline 15 & 41,5 & 24,5 & 138 & 2,98 \\
\hline 16 & 41,5 & 25,5 & 127 & 3,00 \\
\hline 17 & 41,5 & 26,5 & 154 & 3,00 \\
\hline 18 & 41,5 & 27,5 & 164 & 2,73 \\
\hline
\end{tabular}


Таблица 3. Ранжиране по признаците $x$ и $y$

Table 3. Ranking by signs $x$ and $y$

\begin{tabular}{|c|c|c|c|c|c|}
\hline $\begin{array}{l}\text { пореден } \\
\text { номер на } \\
\text { квадрата № }\end{array}$ & $\begin{array}{l}\text { дебелина на } \\
\text { литосферата, } \\
\text { km }(x)\end{array}$ & ранг по $x$ & $\begin{array}{l}\text { пореден } \\
\text { номер на } \\
\text { квадрата № }\end{array}$ & магнитуд (y) & ранг по $y$ \\
\hline 16 & 127 & 1 & 9 & 2,6 & 1 \\
\hline 9 & 134 & 2 & 13 & 2,7 & 2 \\
\hline 15 & 138 & 3 & 5 & 2,73 & 3 \\
\hline 4 & 139 & 4 & 18 & 2,73 & 4 \\
\hline 14 & 139 & 5 & 6 & 2,75 & 5 \\
\hline 2 & 141 & 6 & 11 & 2,81 & 6 \\
\hline 3 & 144 & 7 & 7 & 2,82 & 7 \\
\hline 5 & 149 & 8 & 1 & 2,84 & 8 \\
\hline 17 & 154 & 9 & 14 & 2,84 & 9 \\
\hline 10 & 159 & 10 & 12 & 2,85 & 10 \\
\hline 6 & 160 & 11 & 8 & 2,87 & 11 \\
\hline 1 & 161 & 12 & 2 & 2,88 & 12 \\
\hline 12 & 162 & 13 & 10 & 2,9 & 13 \\
\hline 18 & 164 & 14 & 4 & 2,91 & 14 \\
\hline 13 & 165 & 15 & 3 & 2,92 & 15 \\
\hline 8 & 168 & 16 & 15 & 2,98 & 16 \\
\hline 11 & 176 & 17 & 16 & 3 & 17 \\
\hline 7 & 180 & 18 & 17 & 3 & 18 \\
\hline
\end{tabular}

За коефициента на рангова корелация на Спирмън по формулата (2) е изчислена стойност: $\rho=-0,4097$. За определяне на значимостта на получения коефициент на рангова корелация на Спирмън е използвана таблицата на критичните стойности на коефициента на рангова корелация (Fastovets, Popov, 2012). Ако изчислената стойност на коефициента на ранговата корелация на Спирмън е по-голяма от табличната при съответното ниво на значимост, то се приема хипотезата за значимост на коефициента на рангова корелация. В случая, при $n=18$ и ниво на значимост 0,05 , критичната стойност на коефициента на рангова корелация на Спирмън е равна на 0,399 - изчислената стойност $\rho$ е поголяма от критичната при ниво на значимост $\alpha=0,05$. Следователно, изводът за значимостта на коефициента на рангова корелация на Спирмън при доверителна вероятност $\mathrm{P}=1$ - $\alpha=0,95$, е обоснован. Дебелината на литосферата се корелира с магнитуда на земетресенията с доверителна вероятност $P=0,95$.

Получената регресионна зависимост в резултат на пресмятане на коефициента на рангова корелация на Спирмън може да бъде описана с линейна зависимост: $\mathrm{L}=384-80 \cdot \mathrm{M}$ (фиг. 4). 
Таблица 4. Пресмятане на квадратите на разликите на ранговете

Table 4. Calculating the squares of the rank differences

\begin{tabular}{|c|c|c|c|c|c|c|}
\hline № ח/п & $\begin{array}{c}\text { дебелина на } \\
\text { литосферата } \\
\text { km }(x)\end{array}$ & магнитуд $(y)$ & ранг $x$ & ранг $y$ & $\begin{array}{c}d i(\text { ранг х - } \\
\text { ранг у) }\end{array}$ & $d_{i}^{2}$ \\
\hline 1 & 161 & 2,84 & 12 & 8 & 4 & 16 \\
\hline 2 & 141 & 2,88 & 6 & 12 & -6 & 36 \\
\hline 3 & 144 & 2,92 & 7 & 15 & -8 & 64 \\
\hline 4 & 139 & 2,91 & 4 & 14 & -10 & 100 \\
\hline 5 & 149 & 2,73 & 8 & 4 & 4 & 16 \\
\hline 6 & 160 & 2,75 & 11 & 5 & 6 & 36 \\
\hline 7 & 180 & 2,82 & 18 & 7 & 11 & 121 \\
\hline 8 & 168 & 2,87 & 16 & 11 & 5 & 25 \\
\hline 9 & 134 & 2,6 & 2 & 1 & 1 & 1 \\
\hline 10 & 159 & 2,9 & 10 & 13 & -3 & 9 \\
\hline 11 & 176 & 2,81 & 17 & 6 & 11 & 121 \\
\hline 12 & 162 & 2,85 & 13 & 10 & 3 & 9 \\
\hline 13 & 165 & 2,7 & 15 & 2 & 13 & 169 \\
\hline 14 & 139 & 2,84 & 5 & 9 & -4 & 16 \\
\hline 15 & 138 & 2,98 & 3 & 16 & -13 & 169 \\
\hline 16 & 127 & 3 & 1 & 17 & -16 & 256 \\
\hline 17 & 154 & 3 & 9 & 18 & -9 & 81 \\
\hline 18 & 164 & 2,73 & 14 & 3 & 11 & 121 \\
\hline
\end{tabular}

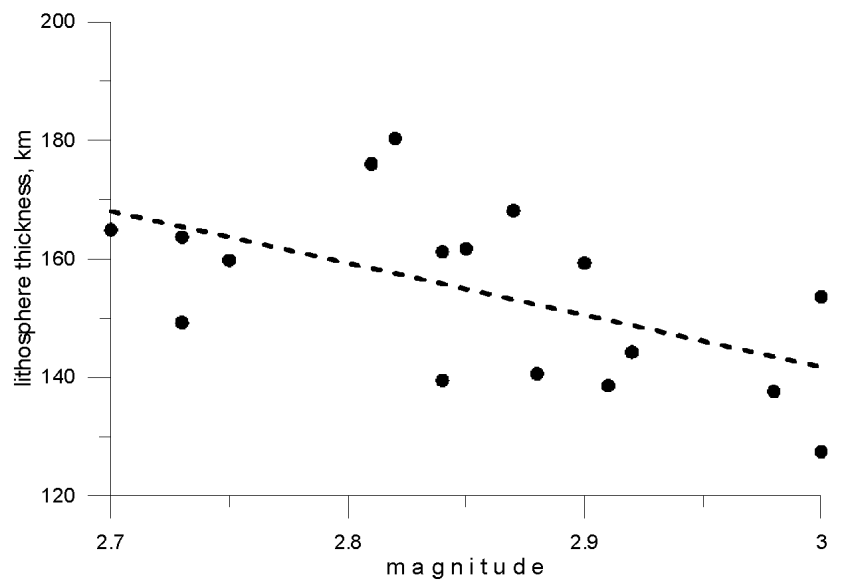

Фиг. 4. Зависимост между магнитуда на земетресенията и дебелината на литосферата в България Fig. 4. Dependence between the magnitude of earthquakes and the thickness of the lithosphere in Bulgaria 


\section{Геотермични предвестници на земетресенията}

Засега са известни множество факти, получени от наблюдения на аномални промени на нивото, дебита и температурата на подземните води, предшестващи и съпровождащи сеизмични явления. Анализът на данните показва многообразието на амплитудите на аномалиите на различни предвестници (от $5 \mathrm{~mm}$ до $57 \mathrm{~m}$ за нивото, от 5 до $500 \%$ за дебита, от 0,5 до $18^{\circ} \mathrm{C}$ за температурата на подземните води), а така също и на времето на проявленията им преди земетресенията (от първи часове до 5-6 години). При повечето случаи амплитудата на ефектите е малка, а най-интензивните колебания се проявяват няколко дни преди земетресение.

Теоретична основа на представата за образуване на хидрогеодинамичните предвестници се явява предпоставката за промяна на напрегнато-деформираното състояние на земната кора преди земетресенията. Съществуващите модели позволяват да се обясни природата на тези предвестници, фиксирани близо до центъра на земетресенията, които се характеризират с наличие на зависимост между магнитуда, времето на проява на предвестника и разстоянието до епицентъра на земетресението (Sobolev, 1984). Природата на предвестниците от 2 клас, при които такава зависимост отсъства, се обяснява с въздействието на съвсем различни фактори: гравитационно притегляне от космически тела, наличие на хидрогеодеформационно поле, наличие на фронт на еластични колебания, а така също и на зони с аномално висока тензочувствителност.

Съществуващите представи за геотермичните ефекти преди земетресение се свеждат основно до два модела: фрикционен и хидродинамичен. Привържениците на първия модел (Kutas, 1978; Ashirov, 1997 и др.) предполагат, че при натрупване на еластични деформации при образуването и развитието на напуканост се осъществява триене по повърхностите на пукнатините, в резултат на което, поради отделяне на фрикционна топлина, по протежението им температурата значително се повишава. В този случай причина за повишаването на температурата може да е и компресията на газовете, запълващи поровото пространство и разтворени в подземните води. Тази гледна точка получи експериментално потвърждение при режимните наблюдения на Ашхабадския сеизмоактивен полигон (Ashirov, 1997). Привържениците на втория модел (Sultanhodzhaev et al., 1978; Lyubimova et al., 1985 и др.) смятат, че по време на преместванията се осъществява интензивен топломасопренос (конвекция) на флуидите по пукнатини. Нахлуването на води от по-дълбоки пластове, имащи, разбира се, по-висока температура, е причина за появата на геотермични аномалии. Проведените режимни наблюдения на термалните води в района на гр. Ташкент, в това число и по време на найсилното разрушително земетресение на 26 април 1966 г. (Geins et al., 1971), на практика потвърдиха геодинамичната природа на температурните аномалии. По всяка вероятност истината, както обикновено, е някъде по средата, и при разработването на теоретични и експериментални основи на прогнозиране на земетресения трябва да се имат в предвид и двата модела.

Анализът на данните от синхронните промени на температурата и дебита на подземни води, получени при силните земетресения в Япония (Takagi et al., 1982), в повечето случаи показва, че знакът на аномалиите съвпада. Това обстоятелство позволява в период на сеизмическа активност да бъде предложен вариант на схемата за едновременни промени на дебита и температурата на подземните води. 


\section{Изводи}

Територията на България се отнася към областта на алпийската нагъвателна фаза, за която е характерна повишена сеизмична активност. По тази причина всякакви теоретически и експериментални проучвания в областта на сеизмичното прогнозиране са актуални и социално значими.

Извършеният анализ на статистическите данни от земетресенията на територията на България и прилежащите земи за 30 годишен период, за топлинния поток и за мощностите на термичната литосфера, показа наличие на генетична или парагенетична връзка на съответните геопараметри. Този факт още веднъж потвърждава необходимостта от комплексно разглеждане на геоложките, хидрогеоложките, геофизичните и геохимичните данни при оценка на сеизмичния риск в активните геодинамични зони.

\section{Благодарности}

Настоящето изследване бе подкрепено финансово от руския „Фонд за фундаментални изследвания“ (проект № 18-55-18004) и българския Фонд „Научни изследвания“ (проект № 02/63-2018, частично проект № ДН 14/1-2017).

\section{Литература \\ References}

Aleksandrova, I., B. Babachkova, E.Botev, B. Dimitrov, B. Delibaltova, C. Dobrev. 1992. Preliminary data on the earthquake in Balkan penisula in July - September 1992. Bulg. Geophys. J., 18, 4, 90-95 (in Bulgarian).

Aleksandrova, I., B. Babachkova, E. Botev, B. Dimitrov, B. Delibaltova, K. Donkova, E. Samardzhieva. 1993. Preliminary data on the earthquake in Balkan penisula in October - Decmber 1992. Bulg. Geophys. J., 19, 1, 71-79 (in Bulgarian).

Ashirov, T. 1997. Geothermy of Kopedag - Caspian and adjacent regions. Ashhabad. Ulum, 371 p. (in Russian).

Botev, E., E. Samardzhieva, B. Dimitrov, B. Babachkova, K. Donkova, I. Alexandrova, B. Delibaltova, S. Velichkova. 1993. Preliminary data on the seismic events recorded by NOTSSI in January - March 1993. Bulg. Geophys. J., 19, 3, 95-105 (in Bulgaria with English abstract).

Botev, E., E. Samardzhieva, B. Dimitrov, B. Babachkova, K. Donkova, I. Alexandrova, B. Delibaltova, S. Velichkova. 1993. Preliminary data on the seismic events recorded by NOTSSI in April - Juny 1993. Bulg. Geophys. J., 19, 4, 145-154 (in Bulgaria with English abstract).

Botev, E., R. Miloshev, B. Dimitrov, B. Babachkova, K. Donkova, I. Alexandrova, B. Delibaltova, S. Velichkova. 1994. Preliminary data on the seismic events recorded by NOTSSI in July - September 1993. Bulg. Geophys. J., 20, 1, 75-83 (in Bulgaria with English abstract).

Botev, E., R. Miloshev, B. Dimitrov, B. Babachkova, K. Donkova, I. Alexandrova, B. Delibaltova, S. Velichkova. 1994. Preliminary data on the seismic events recorded by NOTSSI in October - Decmber 1993. Bulg. Geophys. J., 20, 2, 75-83 (in Bulgaria with English abstract).

Botev, E., R. Miloshev, B. Dimitrov, B. Babachkova, K. Donkova, I. Alexandrova, B. Delibaltova. 1994. Preliminary data on the seismic events recorded by NOTSSI in January - March 1994. Bulg. Geophys. J., 20, 3, 110-119 (in Bulgaria with English abstract).

Botev, E., R. Miloshev, B. Dimitrov, B. Babachkova, K. Donkova, I. Alexandrova, B. Delibaltova, S. Velichkova, E, Samardzhieva. 1994. Preliminary data on the 
seismic events recorded by NOTSSI in April - Juny 1994. Bulg. Geophys. J., 20, 4, 96-106 (in Bulgaria with English abstract).

Botev, E., R. Miloshev, B. Dimitrov, K. Donkova, I. Alexandrova, B. Delibaltova, E. Samardzhieva. 1995. Preliminary data on the seismic events recorded by NOTSSI in July - September 1994. Bulg. Geophys. J., 21, 1, 108-120 (in Bulgaria with English abstract).

Botev, E., R. Miloshev, B. Dimitrov, B. Babachkova, K. Donkova, I. Alexandrova, S. Velichkova, K. Genov.1995. Preliminary data on the seismic events recorded by NOTSSI in October - Decmber 1994. Bulg. Geophys. J., 21, 2, 70-80 (in Bulgaria with English abstract).

Botev, E., R. Miloshev, B. Dimitrov, K. Donkova, B. Babachkova, K. Genov, T. Hristova.1995. Preliminary data on the seismic events recorded by NOTSSI in January - March 1995. Bulg. Geophys. J., 21, 3, 77-88 (in Bulgaria with English abstract).

Botev, E., R. Miloshev, B. Babachkova, K. Genov, B. Dimitrov, K. Donkova, S.Rizhova.1995. Preliminary data on the seismic events recorded by NOTSSI in April - Juny 1995. Bulg. Geophys. J., 21, 4, 96-108 (in Bulgaria with English abstract).

Botev, E., R. Miloshev, B. Babachkova, K. Genov, B. Dimitrov, K. Donkova, S.Rizhova. 1996. Preliminary data on the seismic events recorded by NOTSSI in July - September 1995. Bulg. Geophys. J., 22, 1, 77-82 (in Bulgaria with English abstract).

Botev, E., R. Miloshev, B. Babachkova, B. Dimitrov, K. Genov, K. Donkova, S.Rizhova. 1996. Preliminary data on the seismic events recorded by NOTSSI in October - Decmber 1995. Bulg. Geophys. J., 22, 2, 115-125 (in Bulgaria with English abstract).

Botev, E., R. Miloshev, B. Babachkova, B. Dimitrov, K. Genov, K. Donkova, T.Toteva, I. Tzoncheva. 1996. Preliminary data on the seismic events recorded by NOTSSI in January - March 1996. Bulg. Geophys. J., 22, 3. 65-74 (in Bulgaria with English abstract).

Botev, E., R. Miloshev, B. Babachkova, B. Dimitrov, K. Genov, K. Donkova, I. Tzoncheva, T.Toteva.1996. Preliminary data on the seismic events recorded by NOTSSI in April - Juny 1996. Bulg. Geophys. J., 22, 4. $92-97$ (in Bulgaria with English abstract).

Botev, E., R. Miloshev, B. Babachkova, B. Dimitrov, K. Donkova, I. Tzoncheva K. Genov.1997. Preliminary data on the seismic events recorded by NOTSSI in July - September 1996. Bulg. Geophys. J., 23, 1-2, 136-147 (in Bulgaria with English abstract).

Botev, E., R. Miloshev, B. Babachkova, K. Donkova, I. Tzoncheva K. Genov.1997. Preliminary data on the seismic events recorded by NOTSSI in October - Decmber 1996. Bulg. Geophys. J., 23, 1-2, 148-158 (in Bulgaria with English abstract).

Botev, E., B. Babachkova, K. Donkova, I. Tzoncheva, S. Velichkova, B. Dimitrov, T.Toteva, K. Genov, S. Dimitrova.1997. Preliminary data on the seismic events recorded by NOTSSI in January - June 1996. Bulg. Geophys. J., 23, 3-4, 133-143.

Botev, E., B. Babachkova, B. Dimitrov, S. Velichkova, I. Tzoncheva, K. Donkova, S. Dimitrova. 1998. Preliminary data on the seismic events recorded by NOTSSI in January - June 1997. Bulg. Geophys. J., 24, 1-2, 70-78.

Botev, E., B. Babachkova, B. Dimitrov, S. Velichkova, I. Tzoncheva, K. Donkova, S. Dimitrova, T.Toteva. 1998. Preliminary data on the seismic events recorded by NOTSSI in July - December 1997. Bulg. Geophys. J., 24, 3-4, 204-214. 
Botev, E., B. Babachkova, B. Dimitrov, S. Velichkova, I. Tzoncheva, K. Donkova. 1999. Preliminary data on the seismic events recorded by NOTSSI in July December 1998. Bulg. Geophys. J., 25, 1-4, 204-214.

Botev, E., B. Babachkova, B. Dimitrov, S. Velichkova, I. Tzoncheva, K. Donkova. 2000. Preliminary data on the seismic events recorded by NOTSSI in July December 1999. Bulg. Geophys. J., 26, 1-4, 28-38.

Botev, E., B. Babachkova, B. Dimitrov, S. Velichkova, I. Tzoncheva, K. Donkova, S. Dimitrova. 2002. Preliminary data on the seismic events recorded by NOTSSI in January - June 2001. Bulg. Geophys. J., 28, 1-4, 23-31.

Botev, E., B. Babachkova, S. Velichkova, I. Tzoncheva, B. Dimitrov, K. Donkova, S. Dimitrova. 2003. Preliminary data on the seismic events recorded by NOTSSI in July - December 2001. Bulg. Geophys. J., 29, 74-81.

Botev, E., R. Glavcheva, B. Babachkova, I. Tzoncheva, B., K. Donkova, S. Dimitrova. 2005. Preliminary data on the seismic events recorded by NOTSSI in July December 2002. Bulg. Geophys. J., 31, 1-4, 80-87.

Botev, E., R. Glavcheva, B. Babachkova, S. Velichkova, I. Tzoncheva, K. Donkova, S. Dimitrova. 2007. Preliminary data on the seismic events recorded by NOTSSI in July - December 2003. Bulg. Geophys. J., 33, 1-4, 93-102.

Botev, E., R. Glavcheva, B. Babachkova, S. Velichkova, I. Tzoncheva, K. Donkova, S. Dimitrova. 2009. Preliminary data on the seismic events recorded by NOTSSI in July - December 2004. Bulg. Geophys. J., 35, 1-4, 84-92.

Botev, E., R. Glavcheva, I. Popova, B. Babachkova, S. Velichkova, I. Tzoncheva, S. Dimitrova, G. Georgieva. 2010. Preliminary data on the seismic events recorded by NOTSSI in 2006. Bulg. Geophys. J., 36, 1-4, 99-108.

Botev, E., I. Popova, B. Babachkova, S. Velichkova, I. Tzoncheva, S. Dimitrova, L. Dimitrova. 2011. Preliminary data on the seismic events recorded by NOTSSI in 2008. Bulg. Geophys. J., 37, 73-83.

Botev, E., V. Protopopova, I. Popova, B. Babachkova, S. Velichkova, I. Tzoncheva, S. Dimitrova, V. Boychev, D. Lazarov, P. Raykova. 2012. Preliminary data on the seismic events recorded by NOTSSI in 2011. Bulg. Geophys. J., 38, 34-43.

Botev, E., D. Solakov, L. Christoskov. 2013. On the monitoring of the seismic activity in the territory of Bulgaria and surroundings. Bulg. Geophys. J., 39, 59-68.

Fastovets, N., M. Popov. 2012. Mathematical statistics. Moskow, Oil and gas, 99 p. (in Russian).

Geins, V., A. Grabennikov, G. Kulikov, N. Pichugin, H. Tulyaganov, N. Hodzhabaev. 1971. The results of studying the regime of thermal waters in the area of Tashkent in connection with the Tashkent earthquake. - In: Ulomov V. (ed.) Tashkent earthquake 26.04.66. Tashkent, Fan, 200-208 (in Russian).

Hosney, H. 2000. Geophysical parameters and crustal temperatures characterizing tectonic and heat flow provinces of Egypt. ICEHM, Cairo University, 152-166.

Khutorskoy, M., V. Zvolonskiy, A, Rasskazov. 1999. Monitoring and forecasting of geophysical processes and natural disasters. Moscow, RUDN, 233 p. (in Russian).

Khutorskoy, M., E. Botev, E. Tevereva. 2019a. Correlation between seismicity and heat flow in Bulgaria. Monitoring, 2, 6-13 (in Russian with English abstract).

Khutorskoy, M., N. Kostov, V. Hristov, A. Benderev. 2019b. Heat flow and depth temperatures in the Earth crust of Bulgaria based on numerical modelling. Rew. of Bulg geol. Soc., 80, 2, 443-452 (in Bulgarian with English abstract).

Kutas, R. 1978. Heat flows field and thermal model of the earth $s$ crust. Kiev, Naukova dumka, 147 p. (ìn Russian). 
Lyubimova, E., A. Muhtarov, T. Ismail-Zade. 1985. Temperature variations in the Bilanjik well (Azerbaijan) during the seismic activity of the region. Bul. of $A N$ USSSR. Physics of Earth, 4. 104-108 (in Russian).

Rybach, L., G. Buntebarth. 1984. Thevariation of heatgeneration, density and seismic velocity with rock type in the continental lithosphere. Tectonophysics, 103, 335344.

Sobolev, G. 1984. Harbingers of seismic phenomena. Earthquake Forecast, 4, 120 137 (in Russian).

Solakov, D., S. Simeonova (eds.). 1993. Bulgaria catalogue of earthquakes 19811990. Geophys. Inst. Seismolog. Department, Bulg. Acad. Sci., Sofia, 37 p.

Sultanhodzhaev, S., G. Azizov, T. Zakirov, N. Luneva, N. Saidkamalov, S. Husamiddinov. 1978. Hydrogeo-seismological studies in East Fergana. Tashkent, Fan, 189 p. (in Russian).

Takagi, A., K. Ishibashi, S. Suehiro, T. Usami, T. Matsuda, T. Asada, T. Yoshii, H. Wakita, H. Sato, H. Mizutani. 1984. Earthquake prediction methods. Their use in Japan. Moscow, Nedra, 287 p. (in Russian).

Постъпила: 07.05.2020

Приета: 15.06 .2020
Received: 07 May 2020

Accepted: 15 June 2020 
66 Geological Institute, Bulgarian Academy of Sciences (C) 